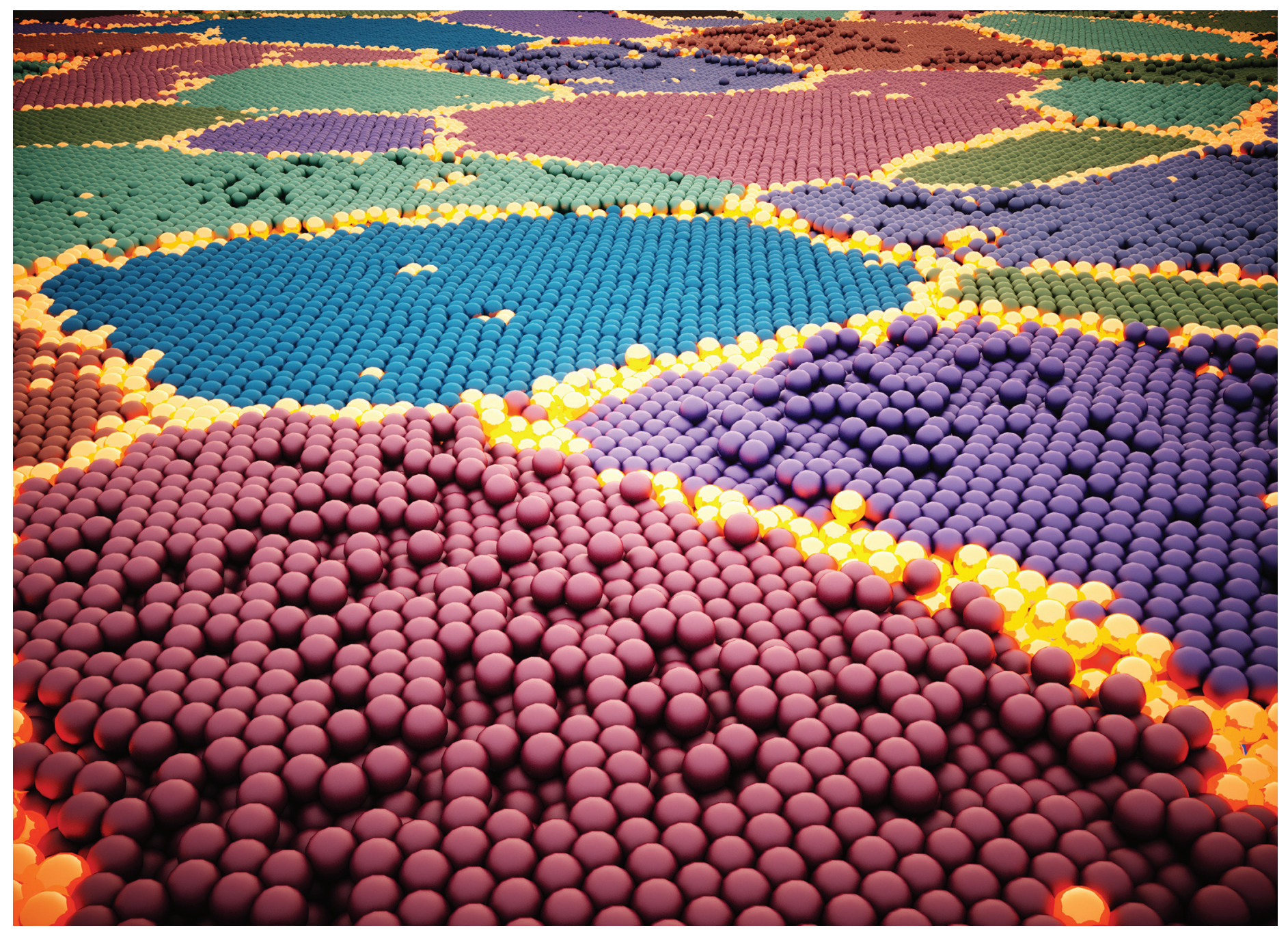

Collaborative research between Prof. Fadi Abdeljawad's group at Clemson University and the Center for Integrated Nanotechnologies at Sandia National Laboratories.

The role of grain boundary character in solute segregation and thermal stability of nanocrystalline Pt-Au

High resolution microscopy, atomistic simulations, and theoretical analysis were used to investigate anisotropic grain boundary (GB) solute segregation and its impact on grain growth and thermal stability of nanocrystalline Pt-Au. Atomistic simulations revealed a plethora of GB segregation profiles as a function of GB misorientation. Theoretical calculations, employing the experimentally obtained GB concentration data were used to elucidate solute drag effects, which were found to vary considerably as a function of GB misorientation.

\section{As featured in:}

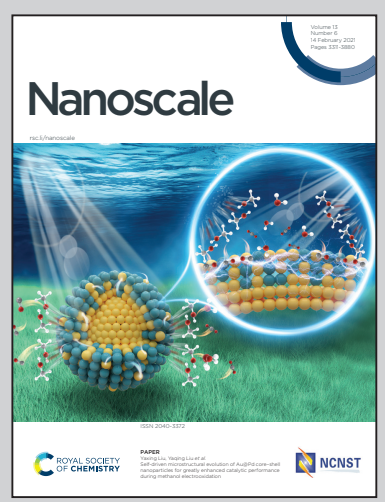

See Fadi Abdeljawad, Khalid Hattar et al., Nanoscale, 2021, 13, 3552. 
(D) Check for updates

Cite this: Nanoscale, 2021, 13, 3552

\title{
The role of grain boundary character in solute segregation and thermal stability of nanocrystalline $\mathrm{Pt}-\mathrm{Au} \uparrow$
}

\author{
Christopher M. Barr, ${ }^{a}$ Stephen M. Foiles, ${ }^{a}$ Malek Alkayyali, ${ }^{\mathrm{b}}$ Yasir Mahmood, ${ }^{\text {b }}$ \\ Patrick M. Price, ${ }^{a}$ David P. Adams, ${ }^{a}$ Brad L. Boyce, (D) a Fadi Abdeljawad (D)*b,c and \\ Khalid Hattar*a
}

\begin{abstract}
Nanocrystalline (NC) metals suffer from an intrinsic thermal instability; their crystalline grains undergo rapid coarsening during processing treatments or under service conditions. Grain boundary (GB) solute segregation has been proposed to mitigate grain growth and thermally stabilize the grain structures of NC metals. However, the role of GB character in solute segregation and thermal stability of NC metals remains poorly understood. Herein, we employ high resolution microscopy techniques, atomistic simulations, and theoretical analysis to investigate and characterize the impact of GB character on segregation behavior and thermal stability in a model NC Pt-Au alloy. High resolution electron microscopy along with $\mathrm{X}$-ray energy dispersive spectroscopy and automated crystallographic orientation mapping is used to obtain spatially correlated Pt crystal orientation, GB misorientation, and Au solute concentration data. Atomistic simulations of polycrystalline Pt-Au systems are used to reveal the plethora of GB segregation profiles as a function of GB misorientation and the corresponding impact on grain growth processes. With the aid of theoretical models of interface segregation, the experimental data for GB concentration profiles are used to extract GB segregation energies, which are then used to elucidate the impact of GB character on solute drag effects. Our results highlight the paramount role of GB character in solute segregation behavior. In broad terms, our approach provides future avenues to employ GB segregation as a microstructure design strategy to develop NC metallic alloys with tailored microstructures.
\end{abstract}

Received 7th October 2020 Accepted 4th January 2021 DOI: $10.1039 /$ dOnr07180c rsc.li/nanoscale counterparts. ${ }^{2,4}$ On the other hand, however, NC metals suffer from a microstructural instability that considerably hinders their deployment in many engineering applications. ${ }^{5,6}$ The increased interfacial contribution to the free energy of NC metals makes them susceptible to rampant grain coarsening and concomitant evolution of their crystal-size dependent properties. $^{7,8}$ The ability to mitigate grain growth in NC materials during processing or under service conditions would enable a myriad of applications ranging from structural and aerospace to energy and electronics. ${ }^{4,9-11}$

In recent years, GB segregation engineering ${ }^{12}$ has been proposed as a design strategy to purposefully dope or enrich GBs with elemental species in order to tailor various interface properties. Enrichment of solutes at GBs has been found to alter many properties of polycrystalline metals, including mechanical strength, ${ }^{13}$ fracture, ${ }^{14}$ wear resistance, ${ }^{11}$ and transport. ${ }^{15}$ The role of GB segregation in grain growth kinetics of NC alloys is of particular interest. Numerous experimental studies have demonstrated enhanced thermal stability in binary NC alloys, including $\mathrm{W}-\mathrm{Ti},{ }^{16} \mathrm{Pd}-\mathrm{Zr},{ }^{17} \mathrm{Pt}-\mathrm{Au},{ }^{18} \mathrm{Y}-\mathrm{Fe},{ }^{19}$ Cu-based, ${ }^{20,21}$ Fe-based, ${ }^{22-25}$ and Ni-based. ${ }^{26-28}$ The preferen- 
tial segregation of solutes to GBs can affect grain growth processes in two main ways. ${ }^{18,29}$ The first is a thermodynamic effect that is described by the Gibbs adsorption equation. The segregation of an alloying element to a GB when the chemical potential is increased at a constant temperature and pressure leads to a reduction in the GB energy, and thus the driving force for grain coarsening. ${ }^{30-32}$ The second is a kinetic mechanism, termed solute drag. ${ }^{33,34}$ Segregated solute atoms will attempt to remain within the migrating GB due to the strong solute atom-GB interactions. As a result, the migrating GB has to drag solute atoms and can only move as fast as the diffusing solutes. In addition to these two mechanisms, if a precipitate is formed by the solute, then the so-called Zener pinning ${ }^{35}$ can also serve to mitigate grain growth. Experimentally, both kinetic and thermodynamic routes act in conjunction, with the relevant role of each depending upon the details of the interactions of solute atoms with GBs, temperature, and solute diffusivity.

While the use of GB solute segregation to mitigate grain growth in NC metals has been the subject of active research, most existing treatments invoke the isotropy assumption in GB segregation behavior, ${ }^{30,32}$ as the dependence of segregation energy on the GB macroscopic geometric degrees of freedom (i.e., three for misorientation and two for plane normal ${ }^{36}$ ) is largely unknown. However, advances in scientific computing and high-resolution experimental techniques have made it possible to investigate in a quantitative manner the anisotropic behavior in many GB properties, including energy, ${ }^{37}$ mobility, ${ }^{38}$ diffusion, ${ }^{39}$ and very recently solute segregation energetics. ${ }^{40-48}$ In one of the earlier studies, Seki et al. ${ }^{40}$ examined Au segregation to [001] twist GBs in Pt using Monte Carlo simulations, and quantified GB concentration profiles as a function of twist angle. Very recently, high throughput atomistic calculations were used to examine the segregation of various elemental species to a wide range of GB types ${ }^{42}$ or to establish GB segregation distributions for specific NC microstructures. ${ }^{43}$ Studies employing high-resolution microscopy ${ }^{18,49}$ and atom probe tomography ${ }^{41,46,47}$ were used to reveal the anisotropy in solute segregation as a function of GB character. While the aforementioned studies have demonstrated that the use of a single segregation energy value to describe solute-GB interactions is an oversimplification, they do not quantify the impact of segregation anisotropy on grain growth processes, nor do they explore the role of segregation anisotropy in the kinetic mechanism (i.e., dynamic solute drag) of NC thermal stability. As suggested by solute drag models, ${ }^{33,34}$ solute segregation effectively alters the GB mobility; therefore, segregation anisotropy results in a distribution of mobilities of doped GBs in a NC microstructure. The existence of distributions in GB migration rates was found to lead to modifications of texture and microstructure and, as a result, materials properties. ${ }^{50-53}$ Therefore, variations in segregation energy as a function of GB character have to be incorporated in materials design and discovery efforts in order for GB segregation to be used as a strategy to develop thermally stable NC materials with tailored microstructures. ${ }^{42}$
Herein, we explore GB solute segregation behavior as a function of GB character after elevated temperature anneals in a model Pt-Au alloy. Undesirable impurity element segregation has often complicated our ability to deconvolute both routes of thermal stability in NC binary alloys. ${ }^{25,54,55}$ The use of the noble $\mathrm{Pt}-\mathrm{Au}$ system minimizes several practical complexities, such as the presence of oxides, that can potentially confound experimental observations. ${ }^{18}$ In this work, high-resolution microscopy and atomistic simulations were used to reveal and quantify anisotropic Au segregation to Pt GBs. Theoretical calculations using the experimentally-obtained GB concentration profiles were then used to elucidate the dependence of GB segregation energies and solute drag forces on GB misorientation.

\section{B. Methods}

\section{Experimental methods}

Pt and Pt-10 at\% $\mathrm{Au}(\mathrm{Pt}-10 \mathrm{Au})$ thin films with a nominal thickness of $40 \mathrm{~nm}$ were magnetron sputtered onto optically polished salt substrates. Sputter deposition involved a Unifilm PVD300 system having a base pressure of $5 \times 10^{-7}$ Torr. Deposition utilized ultrahigh purity Ar which was controlled at 10 mTorr throughout film growth. Films were subsequently floated off the substrates onto TEM Mo mesh grids and annealed in a custom high vacuum furnace at $500{ }^{\circ} \mathrm{C}$ or $700{ }^{\circ} \mathrm{C}$ for 30 minutes at a base pressure of $5 \times 10^{-5}$ Torr. Automated crystal orientation mapping (ACOM) using precession electron diffraction (PED) was completed on the $500{ }^{\circ} \mathrm{C}\left(\sim 0.3 T_{\mathrm{m}}\right)$ and $700{ }^{\circ} \mathrm{C}\left(\sim 0.46 T_{\mathrm{m}}\right)$, where $T_{\mathrm{m}}$ is the melting temperature, annealed Pt and Pt-10Au samples in a JEOL 2100 TEM with a $2.5 \mathrm{~nm}$ step size and $0.375^{\circ}$ precession angle. Orientation maps were post-processed and analyzed in EDAX OIM® 8.1 software using a single pass grain dilation protocol $\left(3^{\circ}\right.$ grain angle criterion). Boundaries were classified as high angle GB (HAGBs) ( $\geq 15^{\circ}$ misorientation) and low angle GBs (LAGBs) $\left(\geq 5^{\circ},<15^{\circ}\right.$ misorientation), and $\sum 3$ twin GBs were classified following the Brandon criteria. ${ }^{56}$ Grain size was calculated as an effective grain diameter following ASTM standards where the standard deviation was calculated as the square root of the variance of the mean. Characterization of GB solute segregation in Pt-10Au was performed in a FEI Titan aberration-corrected scanning/transmission electron microscope (STEM) with a 4 detector X-ray energy dispersive spectroscopy (EDS) system. Sequential STEM-EDS and ACOM data were collected to spatially correlate the localized solute segregation and GB misorientation. The Cliff-Lorimer methodology ${ }^{57}$ was used to quantify and convert X-ray intensity to atomic concentration in the binary alloy. Under the deposition conditions, the $40 \mathrm{~nm}$ thick Pt-Au films tended to grow in a columnar manner, providing one grain through thickness and GBs that were perpendicular to the plane of the film ('edge-on'). GBs that were exclusively edge-on to the electron beam with both grains in similar diffraction contrast were quantified in this study. 


\section{Computational methods}

Atomistic simulations employing a recently developed Embedded Atom Method (EAM) interatomic potential for the $\mathrm{Pt}-\mathrm{Au}$ system $^{58}$ were used to elucidate the impact of anisotropic GB solute segregation on the thermal stability and solute partitioning in NC Pt-Au alloys, and reveal preferential sites for Au segregation within the GB regions. The process of generating the atomistic systems is as follows: a polycrystalline atomistic system with a wide range of GB types was generated using the technique outlined in ref. 59, which employs microstructures generated from phase field or Potts models. Then, the pixelated representation was used to construct periodic atomistic thin films with columnar grains, and these grains were assigned random orientations about their common $\langle 111\rangle$ axis, which was aligned with the film out-of-plane axis. The overall size of the atomistic systems was $\approx 1570 \AA \times 790 \AA$ for the in-plane film dimensions and $\approx 30 \AA$ for the out-of-plane thickness, resulting in a total number of atoms of approximately 2.1 million. Once generated, the atomistic systems were heated to $500{ }^{\circ} \mathrm{C}$ over $50 \mathrm{ps}$ and $\mathrm{Au}$ atoms with a nominal composition of 10 at\% were randomly assigned. The above-mentioned approach of generating the atomistic systems reproduces properties such as triple-junction angles much better than may be achieved by the commonly used Voronoi construction. ${ }^{60}$ Further, the NC atomistic systems were intended to be representative of the temperature, Au composition, texture, and grain-size of the experimental microstructures employed in this study.

After the initial construction, the hybrid Monte Carlo (MC)molecular dynamics (MD) scheme by Foiles ${ }^{61}$ and Sadigh et $a$ l. $^{62}$ was used to equilibrate structural aspects and compositional degrees of freedom of the atomistic systems. With such an approach, an MC simulation step was performed with conserved atomic swaps, in which two atoms are randomly selected and if they were different chemical species, their chemical identities were swapped. The swap was then accepted with a Boltzmann probability based on the energy change at the desired temperature. This MC simulation samples the equilibrium canonical ensemble in a closed system with a fixed number of atoms for each species. In this work, each MC-MD cycle included $10^{7} \mathrm{MC}$ steps followed by a $50 \mathrm{ps} \mathrm{MD}$ simulation at the desired temperature using a Nosé-Hoover thermostat ${ }^{63}$ leading to a trajectory in phase space that is consistent with the canonical NVT ensemble. The use of 50 ps of MD after each MC cycle allows for local structural relaxations after swapping the chemical identity of pairs of atoms during MC. ${ }^{58}$ The MC-MD cycle was repeated until the total simulation time was $20 \mathrm{~ns}$. While the $20 \mathrm{~ns} \mathrm{MD}$ and 30-minute experimental anneals cannot be compared in a one-to-one manner due to the disparity in time scales between these two approaches, our goal was to show that both the experimental studies and atomistic simulations exhibit similar trends in terms of enhanced thermal stability and sluggish grain growth. All atomistic simulations were performed using LAMMPS $^{64}$ and an in-house MC code, ${ }^{40,61}$ and visualizations were generated using Ovito. ${ }^{65}$ The common neighbor analysis (CNA) metric, ${ }^{66}$ polyhedral template matching (PTM), ${ }^{67}$ and dislocation extraction algorithm (DXA) ${ }^{68}$ were used to identify the local structural environment for each atom (FCC, BCC, HCP, etc.) and reveal materials defects, such as GBs and dislocation lines.

\section{Results and discussion}

We start by investigating the microstructural evolution of the experimental NC systems. Fig. 1 depicts variations in thermal stability and grain growth kinetics between $\mathrm{Pt}$ and $\mathrm{Pt}-10 \mathrm{Au}$ systems. Fig. 1(a)-(d) shows representative bright-field TEM images for pure $\mathrm{Pt}$ and $\mathrm{Pt}-10 \mathrm{Au}$ microstructures in the as-deposited and after a 30-minute thermal anneal at $500{ }^{\circ} \mathrm{C}$. The average grain size in pure Pt has grown from $15 \pm 5 \mathrm{~nm}$ to $99 \pm$ $58 \mathrm{~nm}$ (i.e., an increase of $560 \%$ ), while the $\mathrm{Pt}-10 \mathrm{Au}$ one has only grown from $14 \pm 7 \mathrm{~nm}$ to $36 \pm 17 \mathrm{~nm}$ after the $500{ }^{\circ} \mathrm{C}$ 30-minute anneal. Representative inverse pole figure (IPF) colored orientation maps for the $500{ }^{\circ} \mathrm{C}$ 30-minute anneal condition for Pt and Pt-10Au are shown in Fig. 1(e) and (f). In the IPF colored orientation maps, GBs are overlaid where boundaries labeled in black, red, and white denote HAGBs, LAGBs, and $\sum 3$ twin, respectively. The grain size distributions for the $500{ }^{\circ} \mathrm{C}$ and $700{ }^{\circ} \mathrm{C} 30$-minute annealed conditions are shown in Fig. $1(\mathrm{~g})$ and (h), respectively. In both the $500{ }^{\circ} \mathrm{C}$ and $700{ }^{\circ} \mathrm{C}$ grain size distributions, Pt grains show a positively skewed multimodal distribution with a large grain size spread while $\mathrm{Pt}-10 \mathrm{Au}$ has a considerably smaller average grain size in both conditions. The GB character distribution for the Pt and Pt$10 \mathrm{Au}$ NC systems after the $500{ }^{\circ} \mathrm{C}$ anneal condition is shown in Table 1 . The Pt-10Au is observed to have over a three-fold increase in twin boundary length fraction compared to pure Pt while both materials systems have a relatively high length fraction of LAGBs between 7 and 10\%. ACOM data provide the opportunity to examine the orientation texture of the films. In this case, an expected (111) fiber texture developed in the Pt and $\mathrm{Pt}-10 \mathrm{Au}$ free standing films after annealing at both $500{ }^{\circ} \mathrm{C}$ and $700{ }^{\circ} \mathrm{C}$ as indicated by the strong single $\langle 111\rangle$ peak in the (111) pole figure plot shown in ESI Fig. $1 . \dagger$

The experimental trends for grain growth kinetics have also been observed in the atomistic simulations. Fig. 2 shows snapshots of the simulated grain structures for the Pt [Fig. 2(a) and (b)] and Pt-10Au [Fig. 2(c) and (d)] systems in the initial state [Fig. 2(a) and (c)] and after $20 \mathrm{~ns}$ of thermal anneal at $500{ }^{\circ} \mathrm{C}$ [Fig. 2(b) and (d)]. A grain labeling algorithm implemented in Ovito $^{65}$ was used to segment and cluster the atomistic systems based on grain orientations, where atoms in each crystal were assigned a unique color. GB and $\mathrm{Au}$ atoms were colored in gray and black, respectively. It can be seen that the grains in the pure Pt system grew at a faster rate than those in the Pt10Au one. Further, as can be seen from Fig. 2(d), Au strongly segregated to Pt GBs, which resulted in sluggish grain growth kinetics in the Pt-10Au system (see ESI $\uparrow$ for a video of the microstructural evolution). The trends observed in Fig. 2 were 
(a) Pt As-Deposited

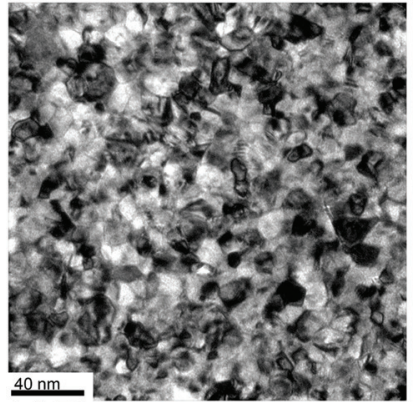

(c) $\mathrm{Pt} 500^{\circ} \mathrm{C}$

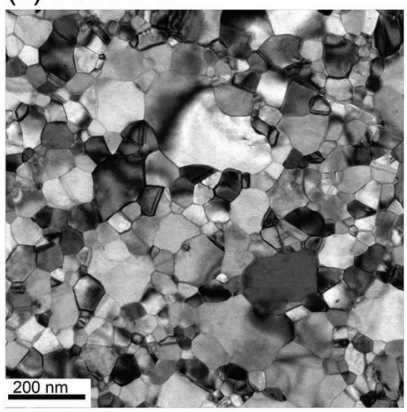

(e) Pt $500^{\circ} \mathrm{C}$ ACOM

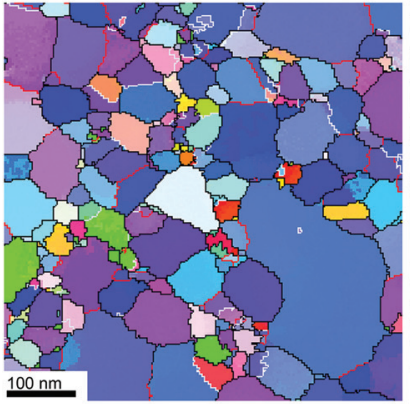

(g)

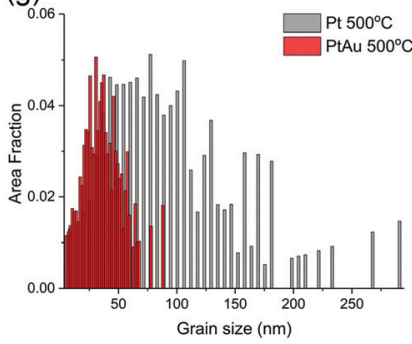

Fig. 1 (a-d) Plan-view bright field TEM images for the ( $a$ and b) as-deposited $\mathrm{Pt}$ and $\mathrm{Pt}-10 \mathrm{Au}$, and ( $\mathrm{c}$ and d) after a 30 minutes anneal at $500{ }^{\circ} \mathrm{C}$. (e and f) Inverse pole figure colored orientation maps for Pt and $\mathrm{Pt}-10 \mathrm{Au}$ after a 30 -minute anneal at $500^{\circ} \mathrm{C}$. (g and h) Grain size distributions for $\mathrm{Pt}$ and $\mathrm{Pt}-10 \mathrm{Au}$ at after 30 -minute anneals at $500{ }^{\circ} \mathrm{C}$ and $700{ }^{\circ} \mathrm{C}$.

Table 1 Overview of the GB length fraction (\%) for high angle, low angle, and $\sum 3$ twin GBs

\begin{tabular}{llcc}
\hline & HAGB $(\%)$ & LAGB $(\%)$ & $\sum 3$ twin $(\%)$ \\
\hline Pt 500 ${ }^{\circ} \mathrm{C}$ & 85.0 & 10.3 & 4.8 \\
Pt-10Au $500{ }^{\circ} \mathrm{C}$ & 76.1 & 7.1 & 16.7
\end{tabular}

quantified by tracking the temporal evolution of both the average grain area $\langle A\rangle$ and number of crystalline grains and the results are shown in Fig. 3. The dashed lines are linear fits to the data using $\langle A\rangle-\langle A\rangle_{\mathrm{o}}=\bar{k} t$, where $\langle A\rangle_{\mathrm{o}}$ is the average grain area in the initial state $(t=0)$ and $\bar{k}$ is a parameter that encompasses several materials properties. It can be seen from Fig. 3 that the pure Pt system is characterized by rapid grain coarsening and concomitant decrease in the number of grains, which was nearly halved after 20 ns of thermal anneal at $500{ }^{\circ} \mathrm{C}$. The Pt-10Au system on the other hand exhibits sluggish growth rates due to the strong Au segregation to Pt GBs. In our atomistic simulations, the fits yielded $\bar{k}=0.60$ and $0.16 \mathrm{~nm}^{2} \mathrm{~ns}^{-1}$ for the pure Pt and Pt-10Au systems, respectively, which indicates a three-fold reduction in growth rates for the Pt-10Au system. Here, we emphasize that one needs to simulate many NC systems with a larger number of grains and various initial grain structures in order to sample all the possible NC configurations and arrive at a dynamical scaling law for the average grain area of doped systems. Nevertheless, by using the linear fit for the average grain area, our goal is to provide a rough estimate of the enhancement in thermal stability due to GB segregation. For the 30-minute thermal anneal of the Pt-10Au system at $700{ }^{\circ} \mathrm{C}$, the average grain size was 103 $\pm 57 \mathrm{~nm}$ compared to $36 \pm 17 \mathrm{~nm}$ for the anneal at $500{ }^{\circ} \mathrm{C}$, refer to Fig. $1(\mathrm{~g})$ and $(\mathrm{h})$. This demonstrates that several factors influencing NC thermal stability, such as Pt-Au solubility and configuration entropy contribution, segregation energy, GB mobility, and solute diffusivity, are sensitively dependent on temperature. ${ }^{18}$

Next, we explore the chemical distribution and solute partitioning in the Pt-10Au system. Fig. 4(a) and (b) shows representative Pt and Au EDS intensity maps for the $500{ }^{\circ} \mathrm{C}$ 30-minute anneal condition, where a non-uniform distribution of Au within individual grains is observed. The non-uniform $\mathrm{Au}$ distribution within the grain interior is attributed to $\mathrm{Au}$ segregation in the immediate vicinity of the GBs and the associated formation of local Au depleted zones; this is further quantified in Fig. 4(c), which shows a line scan over two grains. The line profile indicates both Au depleted zones adjacent to the GBs with an Au concentration nominally below 10 at $\%$ and $\mathrm{Au}$ enriched zones in the center of the grains nominally above 10 at\%. The variation in depth and width of the $\mathrm{Au}$ depleted zones adjacent to GBs is an indication of heterogeneous levels of GB Au concentration. Further, a recent study by O'Brien et al. ${ }^{58}$ demonstrated the existence of a GB phase transformation in $\mathrm{Pt}-\mathrm{Au}$, where low and high solute content regions exist within the GB plane. The experimental trends depicted in Fig. 4 were also observed in the atomistic simulations. Fig. 5 shows snapshots of the atomistic Pt-10Au system after $0.25 \mathrm{~ns}$ [Fig. 5(a)] and $20 \mathrm{~ns}$ [Fig. 5(b)] of thermal anneal at $500{ }^{\circ} \mathrm{C}$ using hybrid MC-MD. The insets show closeup views of a region encompassing several Pt grains. In Fig. 5, FCC Pt atoms were removed for a better visualization of the structures and non-FCC Pt (i.e., GBs) ones were colored in turquoise. Gold and red denote FCC (i.e., in the bulk grains) and non-FCC (i.e., in the GB regions) Au atoms, respectively. In the 

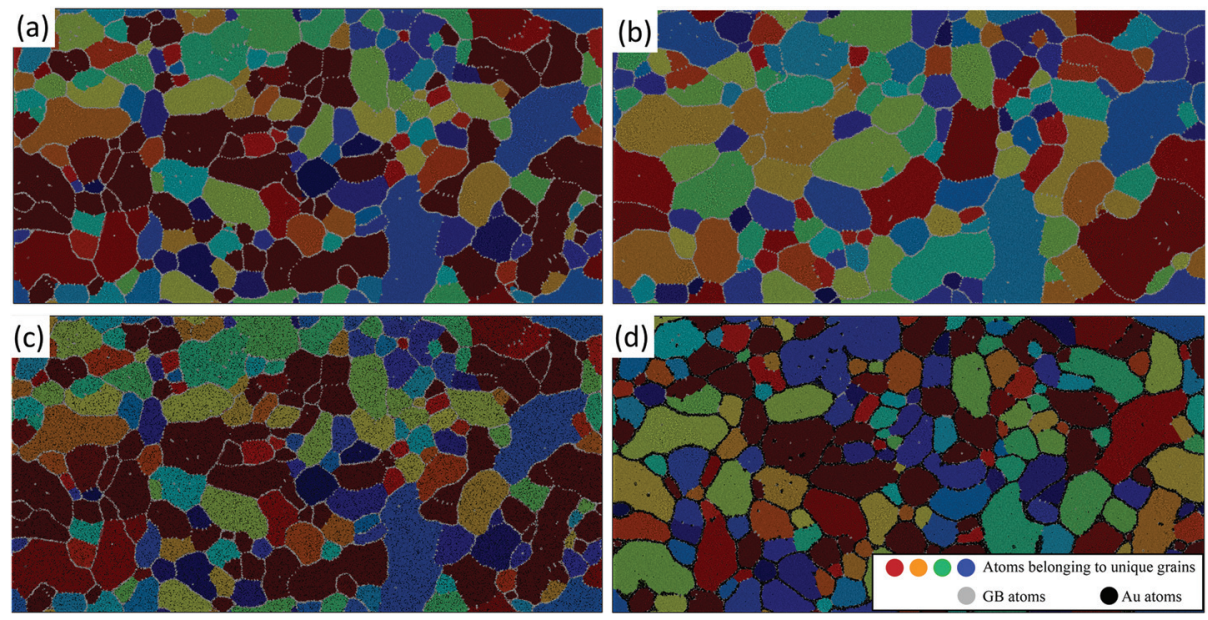

Fig. 2 Snapshots depicting the NC atomistic systems in the $[(\mathrm{a})$ and $(\mathrm{c})]$ initial state and [(b)-(d)] after 20 ns of thermal anneal at $500{ }^{\circ} \mathrm{C}$. The grain structures for the [(a) and (b)] pure Pt and [(c) and (d)] Pt-10Au alloy systems are shown, where atoms in each grain are assigned a unique color.

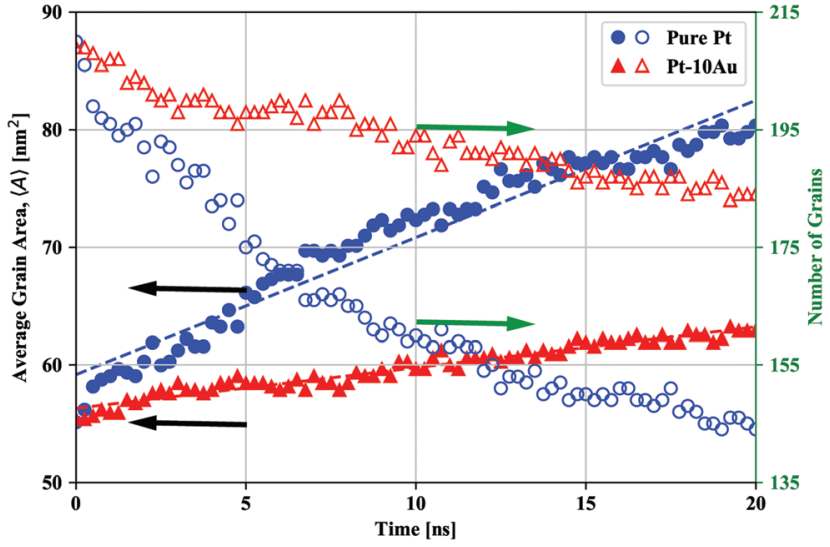

Fig. 3 At a temperature of $500{ }^{\circ} \mathrm{C}$, quantitative analysis of grain growth in the pure $\mathrm{Pt}$ and $\mathrm{Pt}-10 \mathrm{Au} \mathrm{NC}$ atomistic systems depicting the temporal evolution of both the average grain area $\langle A\rangle$ and number of grains. The dashed straight lines are linear fits to the average grain area.

early stage of thermal anneal, both Au phase separation in the form of nanoclusters in Pt grains [cf. Fig. 5(a)] and Au segregation to Pt GBs were observed. This demonstrates competing effects between GB segregation and bulk phase separation, as the Pt-Au alloy is immiscible at this annealing temperature and nominal concentration. ${ }^{69}$ In a phase field study, Abdeljawad et $a l^{70}$ showed that solute partitioning in $\mathrm{NC}$ binary alloys is highly dependent on the relative strengths of GB segregation and bulk heat of mixing energies. Murdoch and Schuh $^{71}$ derived analytical expressions combining GB segregation and heat of mixing energies in order to obtain nanostructure stability and solute distribution maps.

To quantify the level of Au segregation to Pt GBs and its dependence on the GB geometric degrees of freedom, Fig. 6 shows the experimentally-observed GB Au concentration as a function of GB misorientation angle. The location of the misorientation axis for each GB is provided in the inset inverse pole figure. Three global trends of Au segregation levels can be observed in the plot: (1) HAGBs have Au segregation between 30 and 43 at\%; (2) LAGBs show Au enrichment between 17 and 22 at\%; and (3) coherent $\sum 3$ GBs have no detectable Au segregation above the nominal alloy concentration. For example, Fig. 6(b) and (c) shows characteristic Au concentration line profiles for a HAGB and LAGB, respectively. There was no observable trend between Au segregation levels and the misor-
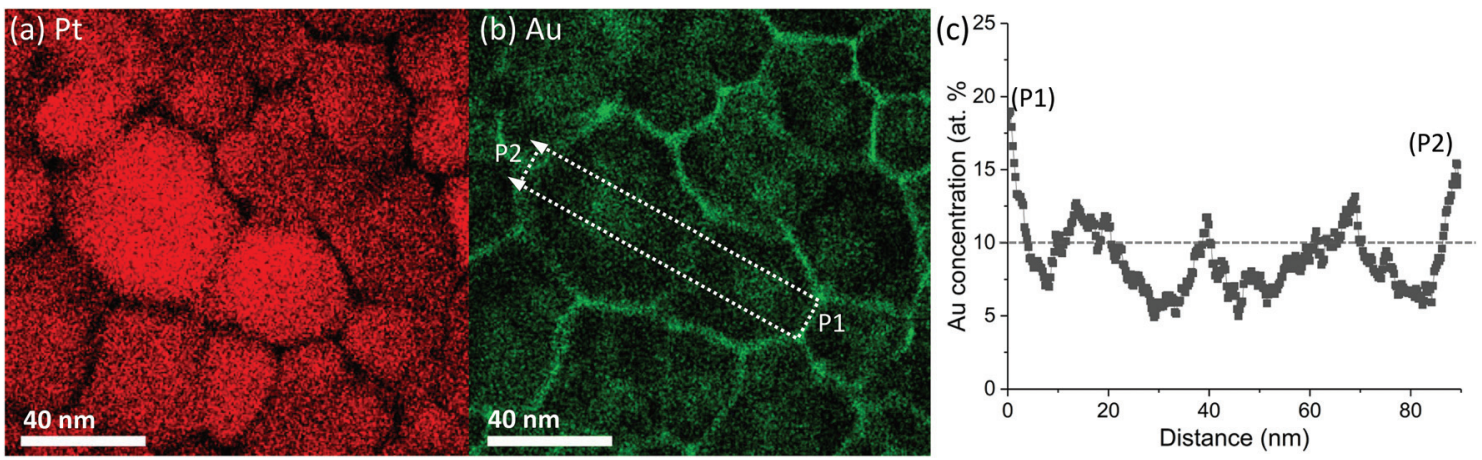

Fig. 4 At a temperature of $500{ }^{\circ} \mathrm{C}$, (a) and (b) Pt and Au X-ray intensity maps where dashed box in (b) indicates the location of the Au concentration line scan shown in (c). 
(a)
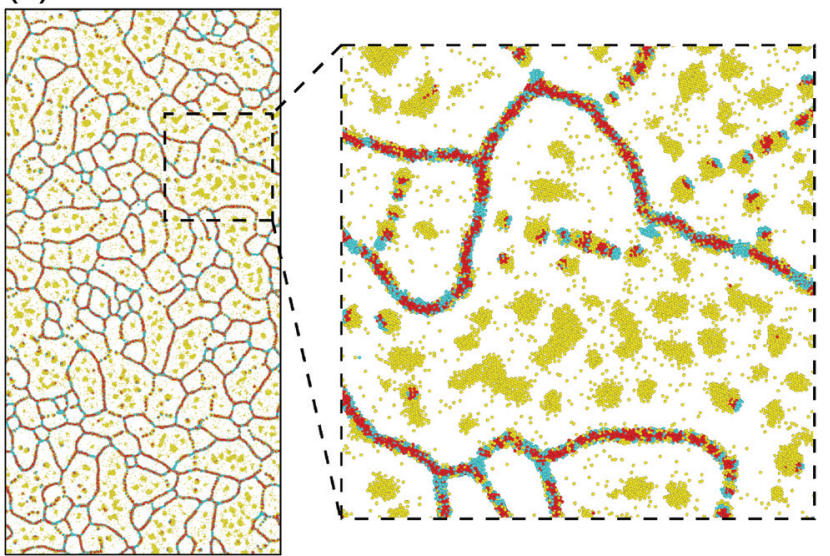

(b)

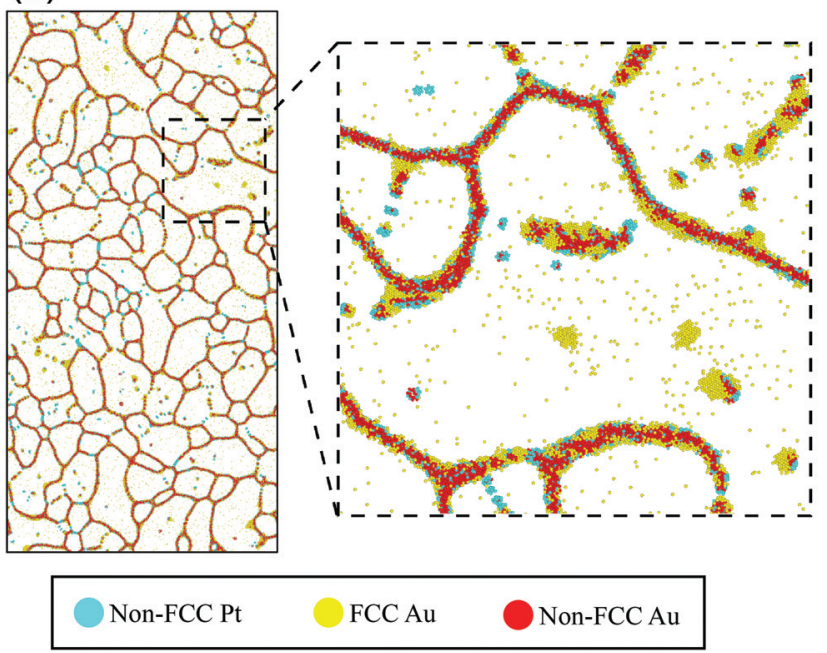

Fig. 5 For the Pt-10Au NC atomistic system, snapshots depicting the temporal evolution of Au after (a) 0.25 ns and (b) 20 ns of MC-MD simulations at $500{ }^{\circ} \mathrm{C}$. The insets show close-up views of a region where $\mathrm{Au}$ phase separates in the bulk Pt grains during the initial stages of thermal annealing then continues to segregate to GBs at late stages. FCC Pt atoms are deleted for a better visualization of the structures.

ientation axis (full GB misorientation data point is shown in Fig. 6(a) inset). The high variation in Au enrichment within the random HAGBs without an apparent trend in misorientation angle could indicate the importance of describing the full macroscopic (i.e., plane normal) and microscopic degrees of freedom defining the GB.

To demonstrate the plethora of $\mathrm{Au}$ segregation profiles present in the Pt-10Au system, Fig. 7 shows snapshots of the atomistic system depicting Au segregation to several Pt GBs after 0.25 ns [Fig. 7(a) and (b)] and 20 ns [Fig. 7(c) and (d)] of thermal anneal at $500{ }^{\circ} \mathrm{C}$ using hybrid MC-MD. Pt FCC atoms in each crystal were assigned a unique color (grey, green, and blue) and non-FCC Pt (i.e., GBs) and Au atoms were colored in turquoise and red, respectively. The blue lines were drawn to guide the eye to grain orientations. For the LAGBs shown in Fig. 7(a) and (b), it can be seen that Au segregates strongly to the dislocations, identified in Ovito using DXA, that make up these GBs; an effect that bears resemblance to the Cottrell atmosphere. $^{72}$ In contrast, Fig. 7(c) shows a symmetric GB, where it can be clearly seen that this boundary does not favor Au segregation. Further, Fig. 7(d) shows a segment of a curved GB (i.e., fixed misorientation but varying plane normal) demonstrating the strong dependency of Au segregation on the GB plane normal. Visual inspection shows no Au segregation to the symmetric segment of this GB while strong Au segregation was observed for the asymmetric part. The experimental observations [Fig. 4 and 6] and atomistic simulation results [Fig. 5 and 7] highlight that not only the misorientation angle, but also the additional GB geometric degrees of freedom, such as plane normal, influence Au segregation. For example, recent studies ${ }^{73-75}$ have shown GB faceting instabilities, in which a macroscopically flat GB breaks into nanoscale facets, and that solute segregation influences this GB faceting behavior. ${ }^{76}$

We now shift our attention to extracting GB segregation energies from the experimental concentration profiles. This GB thermodynamic data serve as input parameters for mesoscale models of GB solute segregation and grain growth kinetics in NC alloys. With the aid of the regular solution approximation, the GB and bulk grain chemical free energies are described as

$$
\begin{aligned}
f_{\mathrm{i}}= & G_{\mathrm{i}}^{\mathrm{Au}} \mathrm{c}+G_{\mathrm{i}}^{\mathrm{Pt}}(1-c)+R T[c \ln c+ \\
& (1-c) \ln (1-c)]+\Omega_{\mathrm{i}} c(1-c), \quad \mathrm{i}=\mathrm{gb}, \mathrm{b}
\end{aligned}
$$

where $c$ is the Au concentration, $G^{\mathrm{Au}}$ and $G^{\mathrm{Pt}}$ are the free energies of pure states, and $\Omega$ is the heat of mixing. $R$ and $T$ are the ideal gas constant and absolute temperature, respectively, and the subscript i indicates a GB or bulk property. Here, the bulk $\Omega_{\mathrm{b}}$ and GB $\Omega_{\mathrm{gb}}$ heat of mixing parameters are assumed concentration-independent. However, one could follow the treatment by Okamoto and Massalski ${ }^{69}$ and allow both $\Omega_{\mathrm{b}}$ and $\Omega_{\mathrm{gb}}$ to be functions of concentration and temperature. Following the treatments outlined in ref. 70 and 77 , the equilibrium GB solute concentration is obtained using the parallel tangent construction, which leads to the following Langmuir-McLean isotherm $^{78,79}$

$$
\frac{c_{\mathrm{gb}}}{1-c_{\mathrm{gb}}}=\frac{c_{\infty}}{1-c_{\infty}} \exp \left[\frac{-\Delta H_{\mathrm{seg}}}{R T}\right],
$$

where $c_{\mathrm{gb}}$ and $c_{\infty}$ are the Au concentration at the center of the GB and bulk nominal concentration, respectively. $\Delta H_{\mathrm{seg}}=\left[\left(G_{\mathrm{gb}}^{\mathrm{Pt}}\right.\right.$ $\left.\left.-G_{\mathrm{b}}^{\mathrm{Pt}}\right)-\left(G_{\mathrm{gb}}^{\mathrm{Au}}-G_{\mathrm{b}}^{\mathrm{Au}}\right)\right]+\Omega_{\mathrm{gb}}\left(1-2 c_{\mathrm{gb}}\right)-\Omega_{\mathrm{b}}\left(1-2 c_{\infty}\right)$ is the GB segregation energy. Based on eqn (1) and (2), changing the temperature modifies the bulk solubility of the $\mathrm{Pt}-\mathrm{Au}$ system and the configuration entropy contribution, and both these effects influence GB concentration profiles. ${ }^{32,70}$ Using GB solute concentration $c_{\mathrm{gb}}$ values from the experimental concentration line profiles obtained through GB edge-on STEM-EDS and PED-ACOM analysis, the above segregation isotherm equation can be used to obtain $\Delta H_{\text {seg }}$ values, and the results are shown in Fig. 8 as function of misorientation angle and axis. It can be seen that the segregation energy increases with 

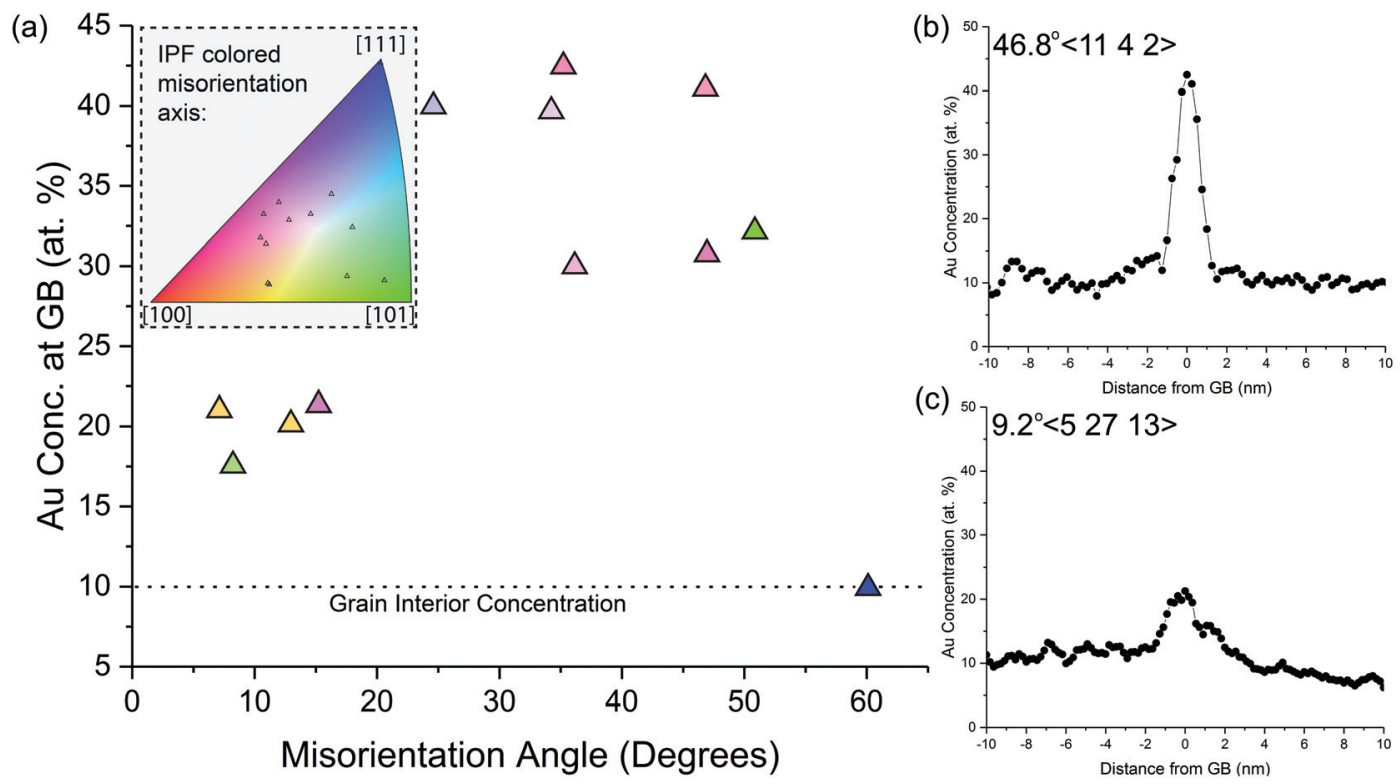

Fig. 6 At a temperature of $500{ }^{\circ} \mathrm{C}$, (a) experimental GB Au concentration as a function of $\mathrm{GB}$ misorientation, where inset inverse pole figure map shows location of misorientation axis for individual GBs identified. A Representative Au concentration profile across a (b) HAGB and (c) LAGB.

the misorientation angle and values for $\Delta H_{\text {seg }}$ ranged between -12 to $-4 \mathrm{~kJ} \mathrm{~mol}^{-1}$. Further, the $\sum 3$ (111) twin GB is characterized by a zero heat of segregation indicating that there is no energetic gain to Au segregation to this GB. As detailed by Lejček and Hofmann, ${ }^{80}$ general trends in GB segregation include increased solute segregation with misorientation angle with specific high index symmetric tilt GBs (e.g., $\sum 3$ (111) and $\sum 11$ (113) in FCC systems) exhibiting low to no segregation behavior. Such trends are also observed in the Pt-10Au system, which show a pronounced lower level of Au segregation to random low angle GBs compared to high angle GBs. The results depicted in Fig. 8 show that for the experimentally characterized NC Pt-10Au sample, the GB segregation energy distribution is bimodal in nature with peaks centered around low and high misorientation angles.

The high degree of heterogenous Au segregation as a function of the misorientation angle and axis shown in Fig. 8 reveals the important impact of GB character and distribution in NC thermal stability. There has been limited exploration of heterogenous solute segregation in NC alloys due to the challenging nature to determine the local GB character. Herbig et $a l .{ }^{81}$ quantified $\mathrm{C}$ segregation in a nanocrystalline colddrawn pearlite steel utilizing a correlative ACOM-TEM and atom probe tomography method. Analogous to the $\mathrm{Pt}-\mathrm{Au}$ result, similar increase in segregation is observed between LAGB and HAGBs with specific outliers in the HAGB associated with special GBs. In a similar fashion, Zhou et al. ${ }^{45}$ examined solute segregation in a binary $\mathrm{Fe}-8 \% \mathrm{Cr}$ alloy where similar trends between LAGB and HAGB were observed. Recent studies have shown that the propensity for solute segregation is also influenced by the local GB structure, which in turn affects processes such as GB structural transitions. ${ }^{76,82,83}$
The anisotropy in GB segregation also plays a key role in dynamic solute drag and the kinetic mechanism of thermal stability in NC alloys; an effect that has been largely overlooked in the NC literature. For a migrating GB with a velocity $V$, Cahn's solute drag model ${ }^{33}$ predicts a GB concentration profile given by (see ESI $\uparrow$ for details on the derivation)

$$
\begin{aligned}
c(x)= & c_{\infty} V e^{\left[-\frac{E(x)}{R T}-V \int_{x_{0}}^{x} \frac{1}{D(\eta)} \mathrm{d} \eta\right]} \\
& \times \int_{-\infty}^{x}\left\{e^{\left[\frac{E(\zeta)}{R T}+V \int_{x_{0}}^{\zeta} \frac{1}{D(\eta)} \mathrm{d} \eta\right]} \frac{1}{D(\zeta)}\right\} \mathrm{d} \zeta,
\end{aligned}
$$

where $\eta$ and $\zeta$ are dummy integration variables. $E(x)$ and $D(x)$ are the position-dependent solute-GB interaction energy and solute diffusivity across the GB region, respectively. In this work, we assumed the forms $E(x)=-E_{\mathrm{o}} \operatorname{sech}(x / \delta)$ and $D(x)=$ $D_{\mathrm{o}} \operatorname{sech}(x / \delta)$, where $\delta=0.1 W_{\mathrm{gb}}$ and $W_{\mathrm{gb}}$ is the GB width (refer to ESI Fig. $2 \dagger$ for a plot of $E(x)$ and the one used by $\left.\mathrm{Cahn}^{33}\right) . E_{\mathrm{o}}$ is the solute-GB interaction energy, which is obtained from our experimental data, and $D_{\mathrm{o}}$ is the solute diffusivity across the GB region. With these forms for $E(x)$ and $D(x)$, the concentration profile across the GB can be parametrized in terms of $E_{\mathrm{o}}$ and $D_{\mathrm{o}}$ as $c=c\left(x / \delta ; V \delta / D_{\mathrm{o}}, E_{\mathrm{o}}\right)$. For a GB with a given segregation energy $E_{\mathrm{o}}$, one can obtain the concentration profile across the GB in terms of the non-dimensional interface velocity $V \delta / D_{\mathrm{o}}$. In Cahn's original work, the nonlinear terms in eqn (3) above were Taylor expanded in order to obtain limiting solutions in the high and low velocity regimes. Herein, we used the experimentally-obtained segregation energies (Fig. 8) along with the differentiable forms for $E(x)$ and $D(x)$ to carry out the integrations in eqn (3) numerically, and the predicted results are shown in Fig. 9. In Fig. 9(a), a surface plot of the solute concentration profile for the GB with the largest segre- 

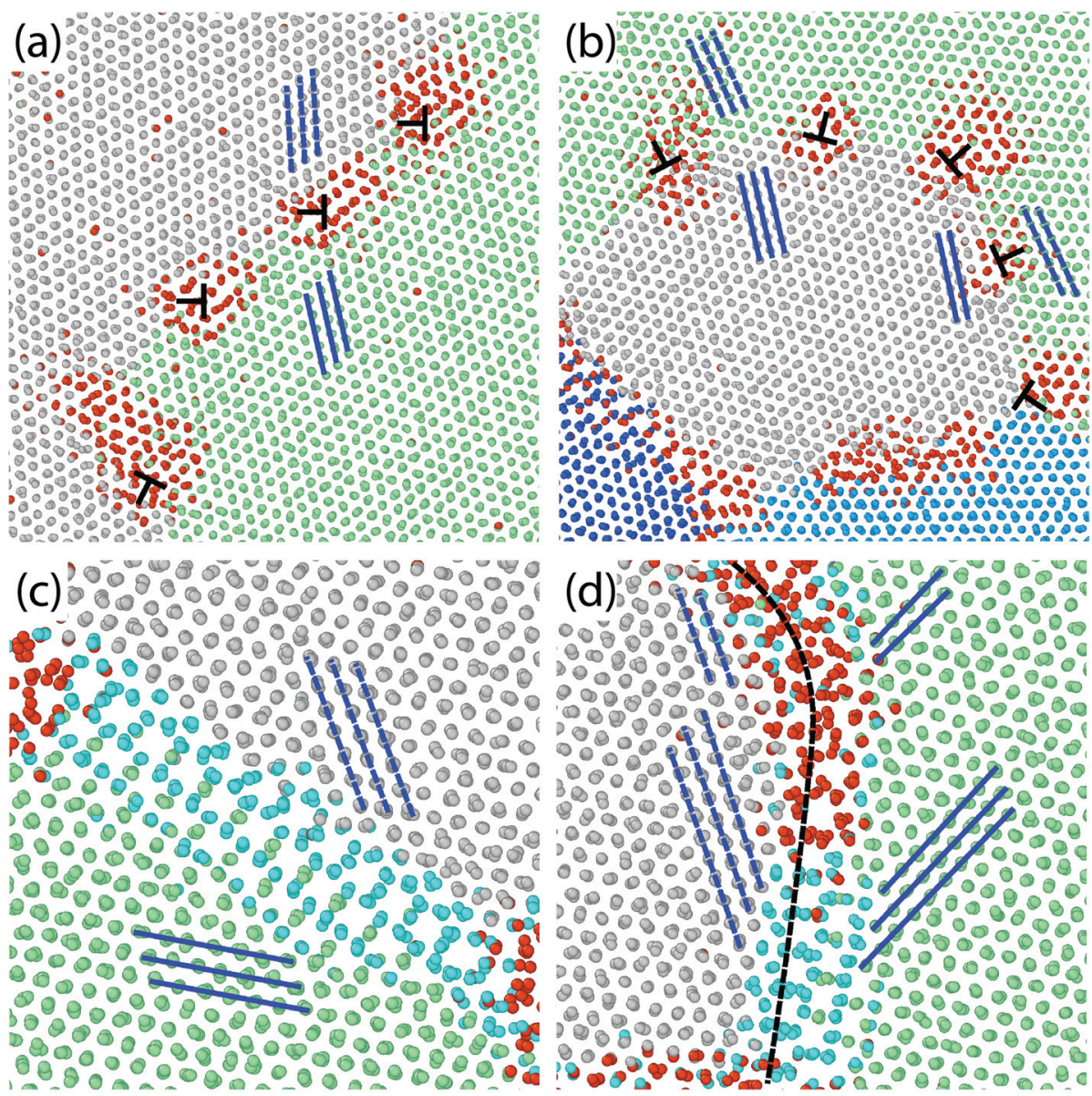

Atoms belonging to unique grains

Non-FCC Pt

$\mathrm{Au}$

Fig. 7 Representative Au segregation profiles to several Pt GBs after [(a) and (b)] $0.25 \mathrm{~ns}$ and [(c) and (d)] 20 ns of thermal anneal at $500{ }^{\circ} \mathrm{C}$. In (a) and (b), Au segregates strongly to dislocation cores that make up LAGBs. (c) A symmetric Pt GB with no Au segregation. (d) Segment of a Pt GB depicting the influence of GB plane normal on Au segregation, where the dashed black line traces the GB profile. The blue lines are drawn to guide the eye to the crystal orientations.

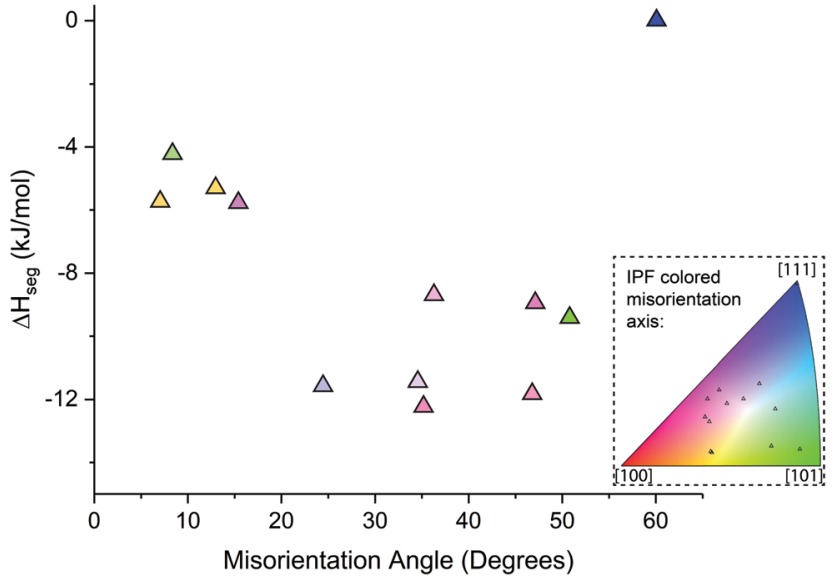

Fig. 8 At a temperature of $500{ }^{\circ} \mathrm{C}, \mathrm{GB}$ segregation energy $\Delta H_{\text {seg }}$ as a function of the GB misorientation angle in the Pt-10Au system. The inset inverse pole figure map shows location of misorientation axis for individual GBs identified. gation energy (misorientation angle of $35.2^{\circ}$ ) is shown as a function of position from the GB center $x / \delta$ and the ratio $V \delta /$ $D_{\mathrm{o}}$. It is evident that the GB concentration profile is highly influenced by the GB migration velocity $V$ and $D_{\mathrm{o}}$. This can be seen from eqn (3) above, the GB segregation energy (thermodynamic effect) and the ratio $V \delta / D_{\mathrm{o}}$ both appear inside the exponential terms, and thus they contribute significantly to the GB concentration profile. To further demonstrate this effect, Fig. 9(b)-(d) shows solute concentration profiles across all experimentally characterized GBs for (b) $V \delta / D_{\mathrm{o}}=0$, (c) $V \delta / D_{\mathrm{o}}$ $=0.1$, and (d) $V \delta / D_{\mathrm{o}}=1.0$. It is clear that GB solute concentration is not only a function of the segregation energy, but a strongly dependent function of the ratio $V \delta / D_{\mathrm{o}}$. As $V \delta / D_{\mathrm{o}}$ increases, as in the case of a large GB migration velocity $V$ in NC alloys and/or slow solute diffusors, GB solute concentration values decrease drastically below ones in stationary GBs, i.e., $V=0$. A close examination of Fig. 9(b)-(d) also shows that the depletion zone in solute concentration ahead of the GB 


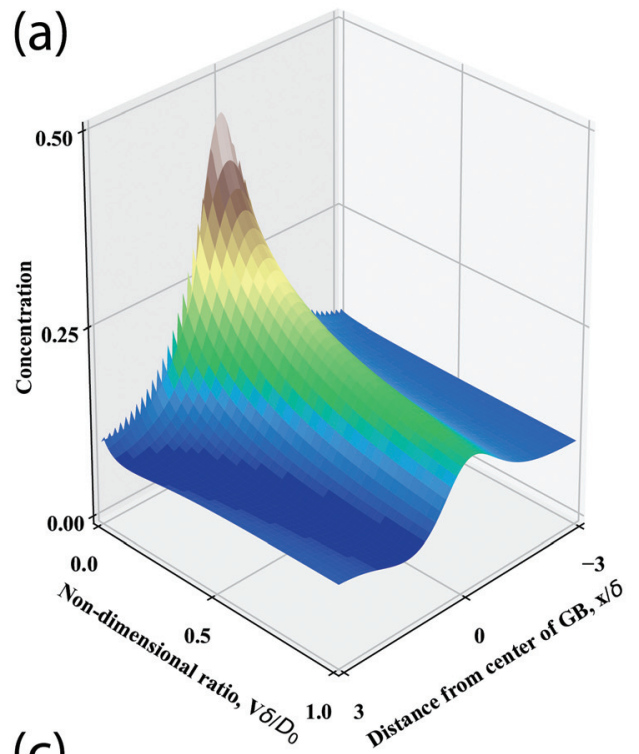

(c)

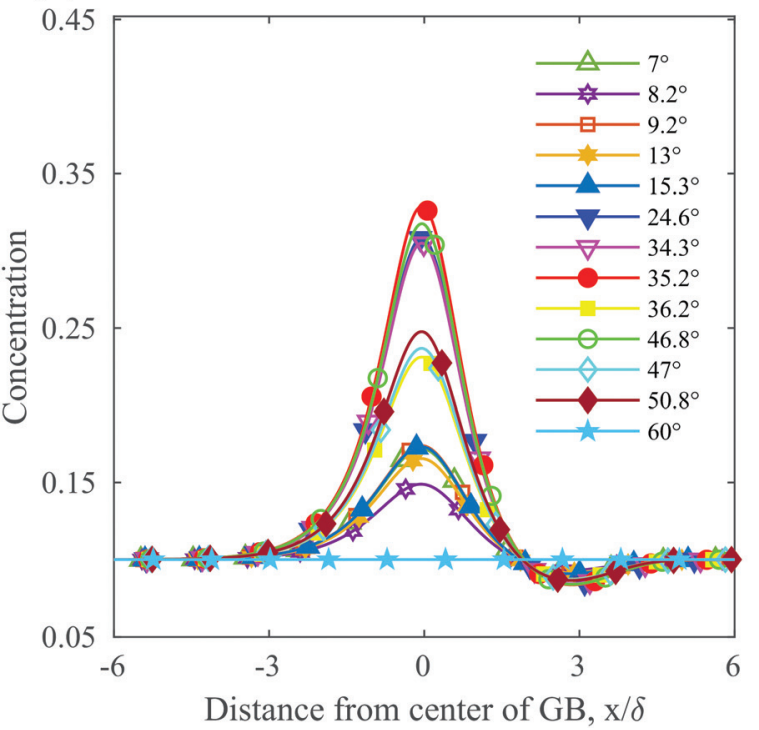

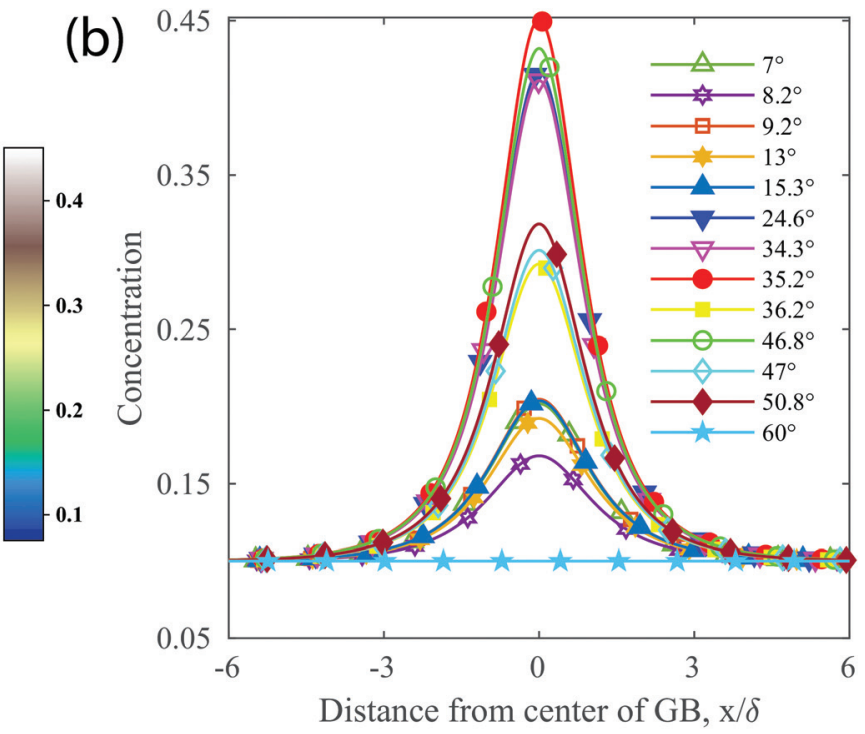

(d)

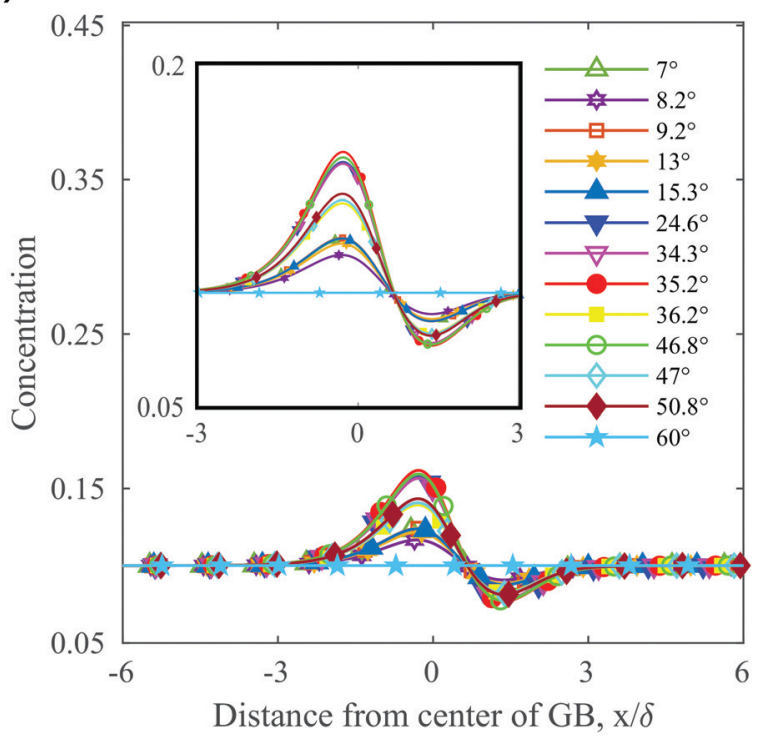

Fig. 9 Theoretical analysis of GB concentrations as a function of the misorientation angle using eqn (3). (a) For the GB given by a misorienation angle $35.2^{\circ}$, a surface plot depicting the boundary concentration as a function of distance from the GB $x / \delta$ and non-dimensional interface velocity $V \delta / D_{\circ}$. (b)-(d) Concentration profiles for all experimentally characterized GBs for $V \delta / D_{\circ}$ values of (b) 0.0 , (c) 0.1 , and (d) 1.0 . The inset in (d) is a close-up view of the concentration profile depicting the asymmetry about the GB center at $x / \delta=0$.

increases in depth as $V \delta / D_{\mathrm{o}}$ increases. Further, the concentration profiles become highly asymmetric about the GB center with increasing $V \delta / D_{\mathrm{o}}$ values.

Finally, the drag force $P_{\text {drag }}$ to the migration of doped GBs is given by ${ }^{33}$

$$
P_{\text {drag }}=\frac{1}{\Omega} \int_{-\infty}^{+\infty}\left(c-c_{\infty}\right) \frac{\mathrm{d} E}{\mathrm{~d} x} \mathrm{~d} x
$$

where $\Omega$ is the molar volume. The concentration profiles in Fig. 9 along with the functional form for $E(x)$ were used to numerically integrate eqn (4) and results for the calculated drag force are shown in Fig. 10 assuming $\Omega=10^{-5} \mathrm{~m}^{3} \mathrm{~mol}^{-1}$.
Fig. 10(a) depicts $P_{\text {drag }}$ as a function of $V \delta / D_{\mathrm{o}}$ for all GBs explored in this work. It can be seen that the drag force increases as the GB starts to migrate until it reaches a maximum, then it decreases sharply with increasing $V \delta / D_{\mathrm{o}}$. The maximum drag force, defined as the peak point in the drag force $-V \delta / D_{\mathrm{o}}$ curve was obtained from Fig. 10(a) and the results are shown in Fig. 10(b) as a function of GB misorientation, where the dashed line is drawn to guide the eye. It can be seen that for the GBs explored in this work the maximum drag force is greatly dependent on GB misorientation. For low misorientation GBs, the drag force is much smaller than high misorientation ones. The coherent twin is characterized by a zerodrag force, as the experimental data show no Au segregation to 
(a)

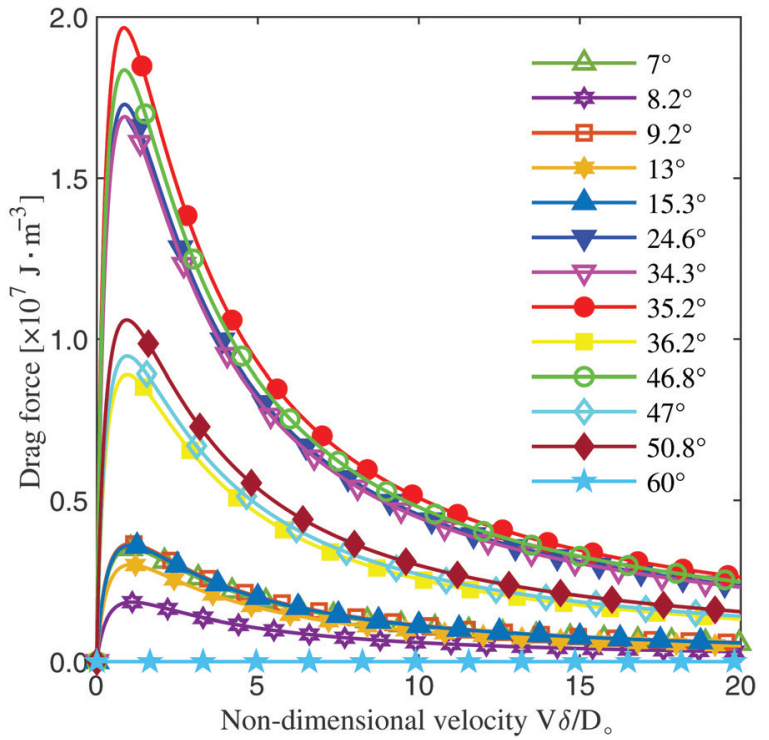

(b)

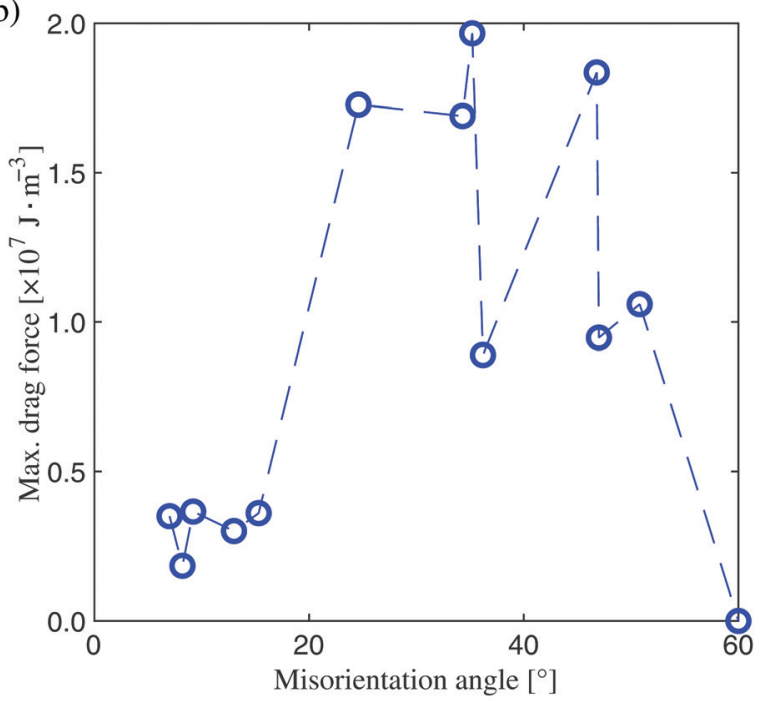

Fig. 10 (a) Using eqn (4), solute drag force as a function of the non-dimensional interface velocity $V \delta / D_{\circ}$ for all GBs explored in this work. (b) The max drag force as a function of GB misorientation, where the dashed blue line is drawn to guide the eye. The max drag force is defined as the peak point in the force $-V \delta / D_{\text {o }}$ curves in (a).

this GB (see Fig. 6). For the experimentally characterized GBs in this work, the calculated maximum drag force to GB migration varies by approximately an order of magnitude.

Our results highlight the need to take into account variations in GB solute segregation as a function of GB character and its role in grain growth kinetics. Previous studies ${ }^{1,52,53}$ have indicated that in pure systems, both GB mobility and energy anisotropy greatly influence grain coarsening kinetics. The results reported in this work show that in solute segregating metallic alloys, both the GB segregation energy and the ratio $V \delta / D_{\mathrm{o}}$ play a critical role in controlling solute drag effects, and that the drag force is greatly influenced by GB misorientation, at least for the GBs explored in this work. Combining both the knowledge of GB character distributions and associated correlations with the level of solute (i.e., GB chemical states) points to a path forward for atomic scale design and engineering of metallic alloys with tailored microstructures by controlling the network and density of solute rich or solute depleted GBs.

\section{Conclusions}

In this work, experimental high-resolution microscopy and automated crystal orientation mapping studies were combined with atomistic simulations and theoretical calculations to quantify GB solute segregation and its dependency on GB misorientation in a model NC Pt-Au system. It was found, using both experimental and computational studies, that GB misorientation greatly influences Au segregation to Pt GBs in a Pt$10 \mathrm{Au}$ alloy. GB concentration profiles and resultant segregation energies varied by up to a factor of 3 as a function of GB misorientation with the coherent twin GB exhibiting no $\mathrm{Au}$ segregation. For the experimentally characterized GBs in our work, theoretical solute drag analysis showed that the drag force for GB motion varied by an order of magnitude as a function of GB misorientation. Further, it was determined that the GB segregation energy alone does not uniquely determine the operating state of a migrating doped GB, as the solute drag force is a highly sensitive function of the ratio of GB velocity to solute diffusivity. Broadly speaking, our approach provides future avenues to use experimentally-obtained GB segregation data in theoretical and computational models in order to quantify the role of GB character in segregation behavior and solute drag effects.

\section{Conflicts of interest}

There are no conflicts to declare.

\section{Acknowledgements}

C. M. B., D. P. A., B. L. B., S. M. F., and K. H. were fully supported by the DOE Office of Basic Energy Science, Materials Science and Engineering Division. This work was performed, in part, at the Center for Integrated Nanotechnologies, an Office of Science User Facility operated for the U.S. Department of Energy (DOE) Office of Science. F. A., M. A. and Y. M. would like to acknowledge financial support from the U. S. Army Research Office through the Young Investigator Program Award (award \#: W911NF-20-2-0122). Sandia National Laboratories is a multimission laboratory managed and operated by National Technology \& Engineering Solutions of Sandia, LLC, a wholly owned subsidiary of Honeywell 
International, Inc., for the U.S. DOE's National Nuclear Security Administration under contract DE-NA-0003525. The views expressed in the article do not necessarily represent the views of the U.S. DOE or the United States Government.

\section{References}

1 E. A. Holm and S. M. Foiles, Science, 2010, 328, 1138.

2 N. Wang, Z. Wang, K. T. Aust and U. Erb, Acta Metall. Mater., 1995, 43, 519.

3 V. Y. Gertsman and S. M. Bruemmer, Acta Mater., 2001, 49, 1589.

4 M. A. Meyers, A. Mishra and D. J. Benson, Prog. Mater. Sci., 2006, 51, 427.

5 R. A. Andrievski, J. Mater., Sci., 2014, 49, 1449.

6 G. Wilde and H. Rösner, J. Mater. Sci., 2007, 42, 1772.

7 M. Saber, C. C. Koch and R. O. Scattergood, Mater. Res. Lett., 2015, 3, 65.

8 M. Saber, H. Kotan, C. Koch and R. O. Scattergood, J. Appl. Phys., 2013, 113, 063515.

9 J. R. Weertman, Science, 2012, 337, 921.

10 H. Gleiter, Acta Mater., 2000, 48, 1.

11 J. F. Curry, T. F. Babuska, T. A. Furnish, P. Lu, D. P. Adams, A. B. Kustas, B. L. Nation, M. T. Dugger, M. Chandross, B. G. Clark, B. L. Boyce, C. A. Schuh and N. Argibay, Adv. Mater., 2018, 30, 1802026.

12 D. Raabe, M. Herbig, S. Sandlöbes, Y. Li, D. Tytko, M. Kuzmina, D. Ponge and P.-P. Choi, Curr. Opin. Solid State Mater. Sci., 2014, 18, 253.

13 V. Yamakov, D. Wolf, S. R. Phillpot and H. Gleiter, Acta Mater., 2003, 51, 4135.

14 O. Dmitrieva, D. Ponge, G. Inden, J. Millán, P. Choi, J. Sietsma and D. Raabe, Acta Mater., 2011, 59, 364.

15 M. R. S. Soares, C. A. R. Costa, E. M. Lanzoni, J. Bettini, C. A. O. Ramirez, F. L. Souza, E. Longo and E. R. Leite, Adv. Electron. Mater., 2019, 5, 1900065.

16 T. Chookajorn, H. A. Murdoch and C. A. Schuh, Science, 2012, 337, 951.

17 B. K. VanLeeuwen, K. A. Darling, C. C. Koch, R. O. Scattergood and B. G. Butler, Acta Mater., 2010, 58, 4292.

18 P. Lu, F. Abdeljawad, M. Rodriguez, M. Chandross, D. P. Adams, B. L. Boyce, B. G. Clark and N. Argibay, Materialia, 2019, 6, 100298.

19 J. Weissmüller, W. Krauss, T. Haubold, R. Birringer and H. Gleiter, Nanostruct. Mater., 1992, 1, 439.

20 T. Frolov, K. A. Darling, L. J. Kecskes and Y. Mishin, Acta Mater., 2012, 60, 2158.

21 M. A. Atwater, D. Roy, K. A. Darling, B. G. Butler, R. O. Scattergood and C. Koch, Mater. Sci. Eng., A, 2012, $558,226$.

22 K. Darling, R. Chan, P. Wong, J. Semones, R. Scattergood and C. Koch, Scr. Mater., 2008, 59, 530.

23 M. Saber, H. Kotan, C. C. Koch and R. O. Scattergood, Mater. Sci. Eng., A, 2012, 556, 664.
24 K. A. Darling, B. VanLeeuwen, J. Semones, C. Koch, R. Scattergood, L. Kecskes and S. Mathaudhu, Mater. Sci. Eng., A, 2011, 528, 4365.

25 B. G. Clark, K. Hattar, M. T. Marshall, T. Chookajorn, B. L. Boyce and C. A. Schuh, JOM, 2016, 68, 1625.

26 B. Färber, E. Cadel, A. Menand, G. Schmitz and R. Kirchheim, Acta Mater., 2000, 48, 789.

27 A. Detor and C. Schuh, Acta Mater., 2007, 55, 4221.

28 K. A. Darling, L. J. Kecskes, M. Atwater, J. Semones, R. O. Scattergood and C. Koch, J. Mater. Res., 2013, 28, 1813.

29 C. Koch, R. Scattergood, M. Saber and H. Kotan, J. Mater. Res., 2013, 28, 1785.

30 J. Weissmüller, Nanostruct. Mater., 1993, 3, 261.

31 J. Weissmüller, J. Mater. Res., 1994, 9, 4.

32 J. Trelewicz and C. Schuh, Phys. Rev. B: Condens. Matter Mater. Phys., 2009, 79, 94112.

33 J. W. Cahn, Acta Metall., 1962, 10, 789.

34 M. Hillert and B. Sundman, Acta Metall., 1976, 24, 731.

35 M. Hillert, Acta Metall., 1988, 36, 3177.

36 D. Rowenhorst, A. D. Rollett, G. S. Rohrer, M. Groeber, M. Jackson, P. J. Konijnenberg and M. De Graef, Modell. Simul. Mater. Sci. Eng., 2015, 23, 083501.

37 D. Olmsted, S. M. Foiles and E. A. Holm, Acta Mater., 2009, 57, 3694.

38 D. Olmsted, E. Holm and S. M. Foiles, Acta Mater., 2009, 57, 3704 .

39 Y. Mishin and C. Herzig, Mater. Sci. Eng., A, 1999, 260, 55.

40 A. Seki, D. Seidman, Y. Oh and S. M. Foiles, Acta Metall. Mater., 1991, 39, 3167.

41 A. Gupta, X. Zhou, G. B. Thompson and G. J. Tucker, Acta Mater., 2020, 190, 113.

42 L. Huber, R. Hadian, B. Grabowski and J. Neugebauer, npj Comput. Mater., 2018, 4, 64.

43 M. Wagih and C. A. Schuh, Acta Mater., 2019, 181, 228.

44 P. Wynblatt and Z. Shi, J. Mater. Sci., 2005, 40, 2765.

45 X. Zhou, X. Yu, T. Kaub, R. L. Martens and G. B. Thompson, Sci. Rep., 2016, 6, 34642.

46 A. Stoffers, O. Cojocaru-Mirédin, W. Seifert, S. Zaefferer, S. Riepe and D. Raabe, Prog. Photovoltaics Res. Appl., 2015, 23, 1742.

47 S. Mandal, K. G. Pradeep, S. Zaefferer and D. Raabe, Scr. Mater., 2014, 81, 16.

48 D. N. Seidman, Annu. Rev. Mater. Res., 2002, 32, 235.

49 J. F. Nie, Y. M. Zhu, J. Z. Liu and X. Y. Fang, Science, 2013, 340, 957.

50 A. D. Rollett, D. J. Srolovitz and M. P. Anderson, Acta Metall., 1989, 37, 1227.

51 G. S. Grest, D. J. Srolovitz and M. P. Anderson, Acta Metall., 1985, 33, 509.

52 A. Kazaryan, Y. Wang, S. A. Dregia and B. R. Patton, Acta Mater., 2002, 50, 2491.

53 M. Upmanyu, G. N. Hassold, A. Kazaryan, E. A. Holm, Y. Wang, B. Patton and D. J. Srolovitz, Interface Sci., 2002, 10, 201. 
54 H. A. Atwater, C. V. Thompson and H. I. Smith, Phys. Rev. Lett., 1988, 60, 112.

55 M. R. He, S. K. Samudrala, G. Kim, P. J. Felfer, A. J. Breen, J. M. Cairney and D. S. Gianola, Nat. Commun., 2016, 7, 1.

56 D. G. Brandon, Acta Metall., 1966, 14, 1479.

57 C. B. Carter and D. B. Williams, Transmission Electron Microscopy: Diffraction, Imaging, and Spectrometry, Springer International Publishing, 2016.

58 C. J. O'Brien, C. M. Barr, P. M. Price, K. Hattar and S. M. Foiles, J. Mater. Sci., 2018, 53, 2911.

59 J. Gruber, H. Lim, F. Abdeljawad, S. Foiles and G. J. Tucker, Comput. Mater. Sci., 2017, 128, 29.

60 G. J. Tucker and S. M. Foiles, Mater. Sci. Eng., A, 2013, 571, 207.

61 S. M. Foiles, Phys. Rev. B: Condens. Matter Mater. Phys., 1985, 32, 7685.

62 B. Sadigh, P. Erhart, A. Stukowski, A. Caro, E. Martinez and L. Zepeda-Ruiz, Phys. Rev. B: Condens. Matter Mater. Phys., 2012, 85, 184203.

63 W. Shinoda, M. Shiga and M. Mikami, Phys. Rev. B: Condens. Matter Mater. Phys., 2004, 69, 134103.

64 S. Plimpton, J. Comput. Phys., 1995, 117, 1.

65 A. Stukowski, Model. Simul. Mater. Sci. Eng., 2010, 18, 015012.

66 J. D. Honeycutt and H. C. Andersen, J. Phys. Chem., 1987, 91, 4950.

67 P. M. Larsen, S. Schmidt and J. Schiøtz, Model. Simul. Mater. Sci. Eng., 2016, 24, 055007.

68 A. Stukowski, V. V. Bulatov and A. Arsenlis, Model. Simul. Mater. Sci. Eng., 2012, 20, 085007.
69 H. Okamoto and T. B. Massalski, Bull. Alloy Phase Diagrams, 1985, 6, 46.

70 F. Abdeljawad, P. Lu, N. Argibay, B. G. Clark, B. L. Boyce and S. M. Foiles, Acta Mater., 2017, 126, 528-539.

71 H. Murdoch and C. Schuh, Acta Mater., 2013, 61, 2121.

72 Y. Mishin and J. W. Cahn, Acta Mater., 2016, 117, 197.

73 F. Abdeljawad, D. L. Medlin, J. A. Zimmerman, K. Hattar and S. M. Foiles, J. Appl. Phys., 2016, 119, 235306.

74 F. Abdeljawad, S. M. Foiles, A. P. Moore, A. R. Hinkle, C. M. Barr, N. M. Heckman, K. Hattar and B. L. Boyce, Acta Mater., 2018, 158, 440.

75 J. C. Hamilton, D. Siegel, I. Daruka and F. Léonard, Phys. Rev. Lett., 2003, 90, 246102.

76 N. J. Peter, T. Frolov, M. J. Duarte, R. Hadian, C. Ophus, C. Kirchlechner, C. H. Liebscher and G. Dehm, Phys. Rev. Lett., 2018, 121, 255502.

77 P. Lejček, Grain Boundary Segregation in Metals, Springer, 2010.

78 D. McLEan, Grain Boundaries in Metals, Oxford Clarendon Press, 1957.

79 E. D. Hondros and M. P. Seah, Metall. Trans. A, 1977, 8, 1363.

80 P. Lejček and S. Hofmann, Crit. Rev. Solid State Mater. Sci., 1995, 20, 1.

81 M. Herbig, D. Raabe, Y. J. Li, P. Choi, S. Zaefferer and S. Goto, Phys. Rev. Lett., 2013, 112, 126103.

82 T. Frolov, M. Asta and Y. Mishin, Phys. Rev. B: Condens. Matter Mater. Phys., 2015, 92, 020103.

83 T. Meiners, T. Frolov, R. E. Rudd, G. Dehm and C. H. Liebscher, Nature, 2020, 579, 375. 